\title{
Infective endocarditis caused by Streptococcus mutans
}

\author{
D. MCGHIE, J. G. P. HUTCHISON, F. NYE, ${ }^{1}$ AND A. P. BALL \\ From the Regional Public Health Laboratory; and the Departments of Communicable and Tropical \\ Diseases, East Birmingham Hospital, Birmingham
}

\begin{abstract}
Members of the viridans group of streptococci are the commonest causes of bacterial endocarditis. However, Streptococcus mutans, a member of this group associated with dental caries which might be expected to be commonly associated with endocarditis, has only rarely been reported. This is possibly because of difficulties in isolation and identification. Differing blood culture media may affect the chances of isolation of these organisms, and, though brain-heart infusion, thiol, tryptic soy, and glucose-brain infusion broths have all proved satisfactory, subcultures may require increased $\mathrm{CO}_{2}$ concentrations for growth. Pleomorphism in the resultant colonies and in the individual organisms may give rise to a hazardous misinterpretation of this appearance as contamination. Strep. mutans and the similarly penicillin sensitive Strep. bovis may be differentiated from the penicillin resistant enterococci by their lincomycin sensitivity and intolerance of 6.3 per cent sodium chloride. Precise differentiation of streptococci in bacterial endocarditis is of value both epidemiologically and in the management of the disease.
\end{abstract}

The viridans group of streptococci are recognised as the commonest organisms associated with infective endocarditis (Cates and Christie, 1951) and remain so despite the recent increase in endocarditis caused by other organisms (Lerner and Weinstein, 1966). The group comprises six main species: Streptococcus sanguis, Strep. mutans, Strep. mitis, Strep. salivarius, Strep. milleri, and Strep.pneumoniae. Strep. mutans is a member of the microflora of the mouth and first achieved significance when described as an aetiological agent in the production of dental caries (Kilian Clarke, 1924). In 1928 Abercrombie and Scott described endocarditis caused by Strep. mutans and recent reports (Harder et al., 1974; Parker and Ball, 1976) indicate that 6 to 14 per cent of cases of streptococcal endocarditis result from infection with this organism. The rarity of reports of isolation of this organism may be accounted for by difficulty in accurately identifying it. Three further cases of Strep. mutans endocarditis occurring in one year in a general hospital are reported here, with a discussion of the cultural characteristics of this organism.

'Present address: Infectious Disease Unit, Fazackerley Hospital, Liverpool L9 78L.

Received for publication 8 November 1976

\section{Case reports}

CASE 1

A 55-year-old man was admitted in January 1974 with a two-week history of fever, dating from a dental scale and polish. He gave a history of rheumatic fever at the age of 18 and of infective endocarditis at the age of 45 , treated successfully with penicillin and streptomycin; he then had signs of mitral regurgitation. On examination, he was febrile $\left(38^{\circ} \mathrm{C}\right)$ and there were subungual splinter haemorrhages. He had atrial fibrillation, signs of moderate mitral regurgitation, and an aortic early diastolic murmur at the left sternal edge. The white blood cell count was $7 \cdot 4 \times 10^{9} / 1$ and ESR $15 \mathrm{~mm}$ in the first hour. Urinalysis showed no abnormality. Strep. mutans was isolated from 9 blood cultures. He was treated with benzylpenicillin $3 \mathrm{M}$ units 6hourly intravenously for 3 weeks, followed by phenoxymethyl penicillin $500 \mathrm{mg}$ 6-hourly by mouth with probenecid for a further 3 weeks, and made a rapid and complete recovery. The patient's serum was bactericidal at dilutions between 1/32 and more than $1 / 512$ on intravenous treatment, and inhibitory at dilutions between $1 / 16$ and $1 / 128$ on oral treatment. The minimum inhibiting concentration of benzylpenicillin was $0.03 \mathrm{~g} / \mathrm{ml}$, and the peak serum level of benzylpenicillin was $18 \mu \mathrm{g} / \mathrm{ml}$. 
CASE 2

A 42-year-old woman was admitted in April 1973 with a 2-week history of fever, myalgia, and ankle oedema. She was known to have a heart murmur which was first detected when she was 25 . On examination, she was febrile, the spleen was enlarged, and there were signs of mitral regurgitation. A digital Osler's node developed a few days later. The white blood cell count was $6.0 \times 10^{9} / 1$ and ESR $58 \mathrm{~mm}$ in the first hour. Urinalysis showed no abnormality. Blood cultures yielded an organism initially thought to be a Corynebacterium, but which was later identified as Strep. mutans. She was treated with benzylpenicillin $1 \mathrm{M}$ units 4-hourly intravenously for 14 days, followed by phenoxymethyl penicillin $1 \mathrm{~g}$ 6-hourly by mouth with probenecid for 10 weeks, and also streptomycin $1 \mathrm{~g}$ daily by intramuscular injection for the first 4 weeks, and made a complete and uncomplicated recovery. The patient's serum was inhibitory to the isolated organism at dilution of $1 / 24$ while she was receiving oral penicillin and streptomycin. The minimum inhibitory concentration benzylpenicillin was 0.12 $\mu \mathrm{g} / \mathrm{ml}$.

CASE 3

A 38-year-old man was admitted in November 1973. He had been ill for 4 months with night sweats, intermittent fever, and weight loss. There was a past history of exemption from National Service on account of a cardiac lesion. He gave no history of rheumatic fever. On examination he was pale and febrile $\left(38.5^{\circ} \mathrm{C}\right)$, and there were signs of mitral regurgitation. The white blood cell count was $9.6 \times 10^{9} / 1$, and ESR $9 \mathrm{~mm}$ in the first hour; urinalysis showed microscopic haematuria. Four blood cultures yielded a growth of Strep. mutans. Treatment with benzylpenicillin $5 \mathrm{M}$ units 6-hourly intravenously with probenecid for 9 days, followed by phenoxymethyl penicillin $500 \mathrm{mg}$ 6-hourly by mouth with probenecid for 4 weeks, resulted in clinical cure. The ESR remained high and phenoxymethyl penicillin $500 \mathrm{mg}$ 6-hourly by mouth was continued for a further 4 weeks. While on intravenous treatment the patient's serum was bactericidal to the isolated Strep. mutans at dilutions between $1 / 1024$ and $1 / 4096$, the minimum inhibitory concentration of benzyl penicillin being 0.007 $\mu \mathrm{g} / \mathrm{ml}$.

\section{Discussion}

The mouth supports an extensive microflora, Streptococcus, Staphylococcus, Veillonella, and Neisseria species being constantly present. Actinomyces, Bacteroides, Corynebacterium, Fusobacterium,
Leptotrichia, and Nocardia species are also commonly found. Despite this wide variety of species, the viridans groups of streptococci appear to be the only oral commensals commonly implicated in the production of infective endocarditis. In their classic study, Cates and Christie (1951) found that this group was responsible for 95 per cent of cases of infective endocarditis. Recently there has been an increase in the number of cases caused by other organisms, notably Staphylococcus aureus and albus (Lerner and Weinstein, 1966), in association with cardiac surgery, renal dialysis, and drug addiction, but the number of cases of viridans endocarditis appears to be little changed. Strep. mutans has been found in 3 cases of endocarditis in a large general hospital in a 10-month period, endorsing the findings of Harder et al. (1974) who reported 9 patients with Strep. mutans endocarditis, representing 6 per cent of all cases of streptococcal endocarditis seen at the Mayo Clinic between 1966 and 1973. In a recent British study of streptococcal endocarditis, there was a similar (14\%) incidence of disease caused by Strep. mutans (Parker and Ball, 1976).

In view of the frequency of these isolations it is relevant to ask why this organism is not being recognised more often. We believe that this is the result of difficulties in isolation and identification of Strep. mutans. Routine blood culture media vary in composition and may not all be suitable for this organism: in case 1 above, though all 9 blood cultures in brain-heart infusion broth yielded Strep. mutans, only 1 of a simultaneous series of cultures in a commercially produced Robertson's cooked meat broth yielded the organism. Glucose-brain infusion broth, tryptic soy broth, and thiol broth have all been found to be satisfactory for the growth of Strep. mutans from blood cultures (Harder et al., 1974) and we have used brain-heart infusion broth successfully. Cultures must be maintained for three weeks and regularly subcultured on to horse-blood agar, in 5 to 10 per cent $\mathrm{CO}_{2}$ and in anaerobic conditions with increased $\mathrm{CO}_{2}$. The dependence of Strep. mutans on $\mathrm{CO}_{2}$ suggests that it should be used in blood culture bottles. Colonies of this organism on blood agar are dry, adherent, and generally nonhaemolytic. At low $p H$ Strep. mutans may be bacillary resembling diphtheroids (Kilian Clarke, 1924), and variation in colonial morphology may lead to a mixed culture appearance. Strep. mutans may be incorrectly identified as an enterococcus, diphtheroid, or lactobacillus, and may be reported as Strep. viridans or a microaerophilic streptococcus. The pleomorphism shown by this organism may result in erroneous interpretation of blood cultures, and disastrous results for the patient may 
Table Differentiation of Strep. mutans and Strep. bovis from enterococci and Strep. mutans from other members of the viridans group

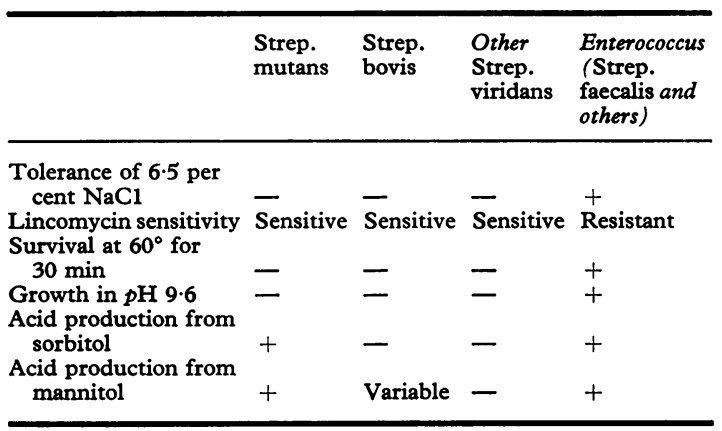

follow if it is thought to be a contaminant and treatment withheld.

Full criteria for the identification of Strep. mutans and details of the test techniques are described in a recent extensive study of the streptococci and aerococci associated with systemic infection in man (Parker and Ball, 1976). Some useful basic features which differentiate Strep. mutans and Strep. bovis from other enterococci, and Strep. mutans from other viridans organisms are shown in the Table. The in vitro sensitivity to lincomycin of the viridans group including Strep. mutans and of Strep. bovis is particularly useful in differentiating them from Strep. faecalis and other enterococci which are highly resistant.

Strep. mutans is found almost exclusively on teeth where it is a major component of dental plaque (MacLean, 1927) and it follows that removal of dental plaque and other minor dental procedures may lead to bacteraemia and subsequent endocardial colonisation by this organism. The factors associated with the colonisation of endocardium by streptococci are largely unknown, but Strep. mutans, in common with Strep. sanguis and Strep. bovis, and some strains of Strep. mitior produce dextran. Elliot (1973) found that dextran was produced by only 9 per cent of streptococci isolated during post-dental extraction bacteraemia but by 34 per cent of streptococci isolated from cases of endocarditis, and suggested that dextran production might promote adhesion of these streptococci to heart valves. Of the 317 cases of streptococcal and aerococcal endocarditis investigated by the Streptococcal Reference Laboratory, 53 per cent were caused by organisms which typically produce dextran (Parker and Ball, 1976).

Precise differentiation of both Strep. mutans and Strep. bovis from other viridans streptococci and from enterococci is worth while, for epidemiological reasons, and also because of the different antibiotic sensitivities of these organisms. Both Strep. mutans and Strep. bovis are invariably sensitive to benzylpenicillin. However, animal studies suggest that a combination of benzylpenicillin and an aminoglycoside results in quicker eradication of even penicillin sensitive viridans organisms from cardiac vegetations (Sande and Irvin, 1974) and this combination is probably indicated as primary therapy in Strep. mutans endocarditis.

We thank Drs. W. M. Phillip, R. Fothergill, and A. M. Geddes for permission to report the above cases.

\section{References}

Abercrombie, G. F., and Scott, W. M. (1928). A case of infective endocarditis due to Streptococcus mutans. Lancet, 2, 697-699.

Cates, J. E., and Christie, R. V. (1951). Subacute bacterial endocarditis. Quarterly fournal of Medicine, 20, 93-130.

Elliot, S. D. (1973). The incidence of Group-H. Streptococci in blood cultures from patients with subacute bacterial endocarditis (SBE). Fournal of Medical Microbiology, 6, p. XIV.

Harder, E. J., Wilkowske, C. J., Washington, J. A., and Geraci, J. E. (1974). Streptococcus mutans endocarditis. Annals of Internal Medicine, 80, 364-368.

Clarke, J. K. (1924). On the bacterial factor in the aetiology of dental caries. British fournal of Experimental Pathology, 5, 141-147.

Lerner, P. I., and Weinstein, L. (1966). Infective endocarditis in the antibiotic era. New England fournal of Medicine, 274, 199-206.

MacLean, I. H. (1927). The bacteriology of dental caries. Proceedings of the Royal Society of Medicine, 20, 873-878.

Parker, M. T., and Ball, L. C. (1976). Streptococci and Aerococci associated with systemic infection in man. fournal of Medical Microbiology, 9, 275-302.

Sande, M. A., and Irvin, R. G. (1974). Penicillin aminoglycoside synergy in experimental Streptococcus viridans endocarditis. Fournal of Infectious Diseases, 129, 572-576.

Requests for reprints to Dr. D. McGhie, Regional Public Health Laboratory, East Birmingham Hospital, Bordesley Green East, Birmingham B9 5ST. 